\title{
Analyzing Enterprise Social Media Networks
}

\author{
Marc Smith, Derek L. Hansen, Eric Gleave
}

\begin{abstract}
Broadening adoption of social media applications within the enterprise offers a new and valuable data source for insight into the social structure of organizations. Social media applications generate networks when employees use features to create "friends" or "contact" networks, reply to messages from other users, edit the same documents as others, or mention the same or similar topics. The resulting networks can be analyzed to reveal basic insights into an organization's structure and dynamics. The creation and analysis of sample social media network datasets is described to illustrate types of enterprise networks and considerations for their analysis.
\end{abstract}

Keywords- Social Network, Enterprise, Social Media, Analysis, Social Graph, Organization

\section{INTRODUCTION}

Social network data sets are generated by many tools, not just those called "social networking services." A rich new source of social network data is created as enterprises and organizations adopt social media tools like message boards, blogs, wikis, friend and contact networks, activity streams and file, photo, and video shares. Enterprise social media applications bring applications found on the public Internet into the confines of an institution's firewall. These tools serve as detailed records of social networks, providing maps of the structure of social connections within an organization. Data captured from social media tools also create networks connecting individuals with artifacts (e.g., digital artifacts) and groups. While social networks have always existed in human institutions, only recently have such richly detailed networks been made available in machine readable format as a natural byproduct of interaction between people and their artifacts.

Extracting, processing, and analyzing these networks can reveal important patterns in the structure and dynamics of the institutions that adopt these tools. Network analysis is the sociological application of mathematical graph theory to data representing sets of relationships. Social network structures are created when people connect to one another through a range of ties. Network analysis is a set of methods for calculating measures that describe a population of "nodes" (representing people and other entities) and their "edges" or relationships to one another. These metrics can describe the overall shape and size of a network as well as describe the location and connection pattern of each node (or vertex) in the network. Tools that visualize networks can reveal additional insights, particularly when they integrate visual properties (e.g., node size or color) with network metrics (e.g., degree, betweenness centrality) $[1,2]$.

When applied to enterprise social media networks, network analysis metrics and visualizations can highlight important people, events, and subgroups within an organization. Each person in the organization is connected to a potentially different number and set of other people. Network metrics illustrate the ways people specialize in different patterns of connection, identifying that person as occupying and performing a particular "role" (or roles) in the organization. Examples of roles include "answer people" [3] who disproportionately provide the answers to questions asked in message board environments, "discussion people" who engage in extended exchanges of messages in large and populous threaded discussions [4], "discussion starters" who demonstrate influence over the topics discussed by the "discussion people" [5], "influential" people who are well connected to others who are more highly connected than they are, and boundary spanners who bridge between unconnected subgroups [6]. The ability to identify individuals within an organization with particular network properties can be applied to improve enterprise search applications, provide better reporting and ranking services, and can be used to guide management by producing reports on the rates of internal connection within and across groups in an organization. Changes over time can also be tracked to better understand the impact of external or internal events such as changes in employee size or makeup.

Following a review of related work, we document a growing number of different social networks being generated as enterprises adopt social media applications. We note the various properties of these networks and describe the construction and application of social network metrics for reporting on the quality, health and leadership of social media spaces. We conclude with a discussion of emerging forms of social network data sets, potential concerns with using this data, and the directions for future work.

\section{RELATED WORK}

Social network analysis has a long history and connection to the study of organizations and businesses. Early social network literature was built on manually collected and processed data about social ties. Researchers would typically observe or survey organization members, asking each to list those they came in contact with regularly for a variety of tasks and purposes. The prohibitive cost of this approach was a major limiting factor in the widespread application of social network analysis in enterprises and organizations. Despite these challenges, a healthy subfield developed around social network analysis of large organizations, as evidenced by the growing body of literature written for business audiences [7-9]. Social network analysis and metrics are described in [10].

Researchers and practitioners in the subfield of enterprise social network analysis have documented the impact social structure has on outcomes of interest to organizations and employees. For example, Cross and colleagues illustrate several practical applications of social network analysis for corporations and other large organizations, highlighting differences between healthy and under-performing divisions and the value of organization spanning connections [7-8]. 
Others like Burt provide compelling evidence that individuals who bridge structural holes are promoted faster than others [6].

Researchers and practitioners have begun to use automatically captured social media data to create and analyze social networks. The everyday use of social media tools for day-to-day activities generates rich network data without having to explicitly ask each employee about their personal connections. Researchers interested in enterprise social networks have begun to explore these networks. For example, Perer and Smith [11] provided a tool that allowed employees to visualize their corporate email collection. The resulting images highlight structures of communication activity across the organizational chart of the corporation. Others have related email usage and network characteristics to individual productivity $[12,13]$. Researchers can now move beyond email and the very real concerns about research use of private communication to take full advantage of the network data created as a byproduct of the use of other more explicitly public enterprise social media tools such as wikis, forums, blogs, microblogs, and status updates.

Although literature on enterprise social media networks is relatively limited, there has been a surge of research that examines social networks based on the use of social media tools in non-enterprise settings. A series of papers have documented the ways contributors to social media repositories (e.g., Usenet) have distinct patterns of contribution and connection to other contributors [3-5]. These patterns are evidence of specialization of behavior in these social spaces.

A separate line of research has focused on providing tools that help make sense of social network data through visualizations. Some tools such as NodeXL automatically extract social media networks and allow users to perform a set of core operations that measure and map the resulting dataset [1]. Other tools developed by Viegas and colleagues use nongraph-based visualizations to make sense of social media data such as email [14] and wikis [15].

Eagle and Macy have been analyzing the "call graph" created when people use their telephones and mobile phones to call one another. Their research on a data set containing all the phone calls in the United Kingdom for a six-week period highlights different patterns of calling. They find that economically prosperous regions display higher diversity of connections than economically marginal regions.

A growing area of research involves the application of mobile or location based sensors to capture evidence of social connections and, by extension, social networks. Sandy Pentland and the SenseNetworks company are building tools that use cell phone towers' ability to collect data from cell phones to map the locations of groups of people over time. The resulting patterns are used to group people into "tribes" based on common, overlapping habits. Even if two people have never met, their common use of certain kinds of spaces and transit systems soon build a link based on their shared visits to the same kinds of restaurants, theaters, office buildings, and highways or rail lines. These connections are examples of what Counts and Smith call "hyperties," or links that are created between people when machines find associations between people based on common locations or activities even if people never meet or occupy the same locations at the same time [16]. The term "hyperties" echoes the attention Goffman gave to "tie signs" that indicated a connection between two people. Hyperties, unlike analog tie signs, are machine authored based on a range of statistical associations and similarities.

To date, analysis of social networks has remained largely an academic endeavor conducted by PhDs trained to apply its specialized concepts and software to answer hypotheses of interest primarily to academics. We envision a time when social network analysis is also used by organizational managers, online community administrators, and interested individuals to inform their decisions about practical real-world problems. For this to become a reality, tools and strategies must be developed that support the complex process of making sense of the many networks that are created by social media tools.

In this paper we provide a framework for characterizing the many types of enterprise social media networks. Understanding these networks is vital in educating future network analysts and in designing tools that support their analysis and visualization. We next discuss methods of analyzing and using that data in the goal of contributing to the development of additional network metrics, analytical tools, and theories that take full advantage of the many novel social media data sources available. We conclude with a review of concerns about the potential misuse of these types of data.

\section{TYPES OF ENTERPRISE SOCIAL MEDIA NETWORKS}

The use of social media generates a number of networks. In most organizations, people adopt a patchwork quilt of social media tools. The use of each tool may generate one or more social networks of interest. Public and private email discussions, message boards, "friends", "buddies", and "contacts", wikis, blogs, and file and photos stores, along with comments and readership records, (among others) all generate graph structures that can be extracted and analyzed through the core methods and measures of social network analysis. The resulting social media networks can vary in important ways. In this section we outline a few important dimensions on which these networks vary including: unimodal/multimodal, direct/indirect, symmetry, and weighted/unweighted.

\section{A. Network Properties Related to Nodes (i.e., Vertices)}

Unimodal networks link only one type of entity (i.e., nodes) together. In standard social network analysis all nodes represent people. However, unimodal networks may connect objects to objects (e.g., wiki pages), collections to collections (e.g., playlists), groups to groups (e.g., corporations), terms to terms, locations to locations, activities to activities, etc. The links of unimodal networks may be based on direct associations (e.g., wiki pages linked together based on hypertext) or indirect associations (e.g., wiki pages linked together based on number of co-editors). A number of standard network metrics such as degree, betweenness centrality, eigenvector centrality, closeness centrality, and measures of network density can be used to characterize these networks.

Multimodal networks are composed of mixtures of entities. For example, people, documents, and companies might all coexist in the same network graph. A common type of multimodal network is a bimodal network that connects people 
to a set of similar objects. An example of a bimodal network is shown in Figure 1, where people are linked to wiki pages they have edited.

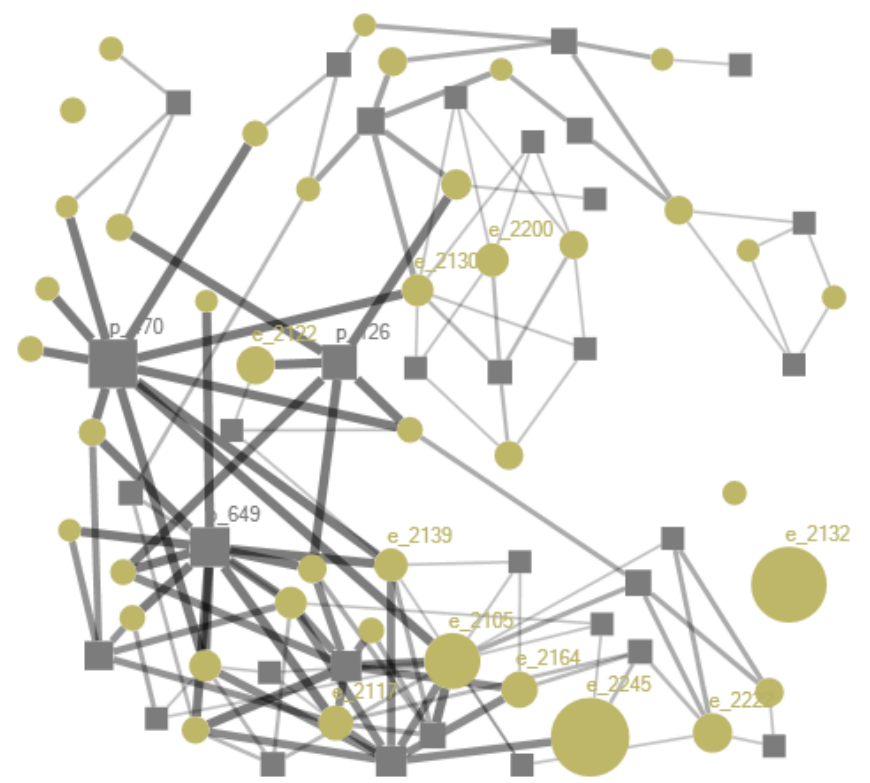

Figure 1. Bimodal Wiki Page and Editor Graph. This bimodal graph shows employees (circles) and wiki pages (squares). Edge thickness indicates number of edits to a page. Node size is based on degree (i.e., large squares have been edited by many people; large circles have edited many pages). Nodes with less than 2 degrees have been removed. Thus, employee e_2132 has a high degree, but only edits pages that nobody else has edited. In contrast, employee 2105 edits pages that are edited by many people.

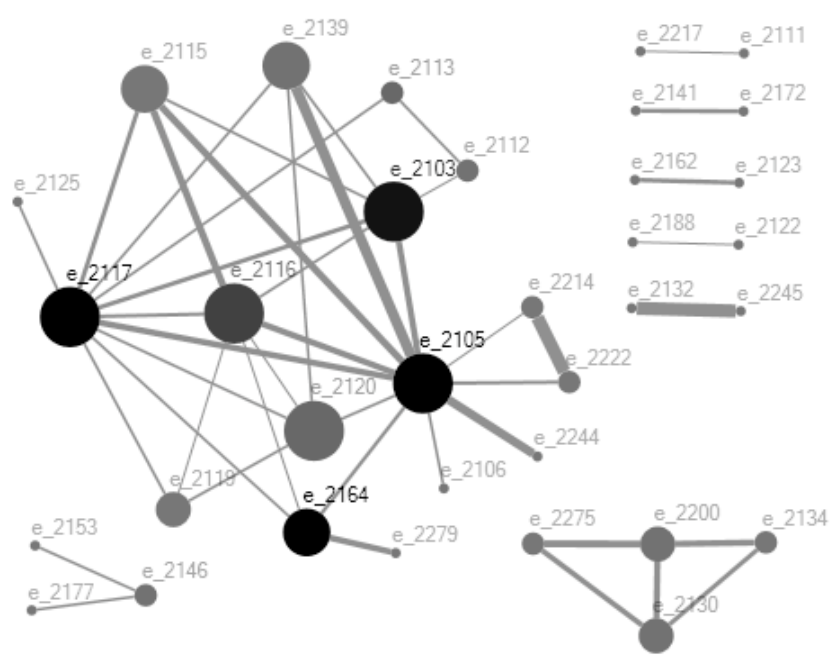

Figure 2. Unimodal Wiki Page Editor Graph. This unimodal graph shows only people. It is based on a transformed version of the data represented in Figure 1. People who have coedited at least 5 of the same wiki pages are connected with an edge. Thicker edges represent more co-edited pages. Node radius is based on degree. Nodes are darker if they have a higher betweenness centrality. Note that e 2105 shows up prominently in this graph, but e 2132 doesn't show up at all, since he only edits pages others have not edited. Thus, each graph highlights different individuals who may play important and distinct roles, as well as their relationship to each other.
Many of the standard network metrics do not apply to multimodal networks (e.g., betweenness centrality), although some apply if appropriately interpreted (e.g., degree) and other, more specialized, metrics have been devised for common types of multimodal networks such as bimodal networks.

Multimodal networks can be reduced to uni-modal networks. For example, "people to document to people" networks can be transformed into "people to people" networks and/or "document to document" networks. An example of a network transformed from a bi-modal to a single mode is shown in Figures 1 and 2. Figure 2 shows the person to person network based on wiki page co-edits. A complementary graph could be created that connects wiki pages to wiki pages based on the number of people who have edited both pages. More generally, this approach can be used to relate objects of all types (e.g., books, photos, audio recordings) based on social network ties rather than content indexes or keyword similarity. Such networks are the raw material for recommender systems: queries that generate the results of "people who linked to this document also linked to these documents" or "if you link to this document, you may want to link to these people".

\section{B. Network Properties Related to Ties (i.e., Edges)}

Networks differ in several ways based on the type of ties that connect nodes to one another, whether the networks are unimodal or multimodal. These distinctions affect the metrics and maps generated from them, as well as their interpretation. Although these graph types are described in social network analysis textbooks, they have not been related to social media network datasets in any systematic way.

Direct graphs describe explicit relationships between entities such as social networking site's ability to friend or follow another person. Indirect graphs describe relationships between entities based on implicit relationships such as the relationship between people based on wiki page co-edits as shown in Figure 2. Another example would be two people connected because they are members of the same group even though they may not know one another.

In symmetric graphs, the relationship tie is reciprocal between the two entities that are connected. For example, in many social networking sites (e.g., Facebook) a Friendship relationship is symmetric since if Person A has "friended" Person B then Person B has also "friended" Person A. In contrast, other networks are asymmetric, suggesting that a tie from Person A to Person B is not necessarily reciprocated. For example, Person A may follow Person B on the Twitter microblogging service without Person B following Person A. Another example is the reply network created in discussion forums and email lists. Asymmetric graphs are often represented using arrows on the edges of a graph.

Weighted graphs allow for the differentiation of edges based on some criteria of interest (e.g., number of messages exchanged or posted). They are often represented visually as thicker or darker lines connecting nodes as was done in Figures 1 and 2. All edges in an unweighted graph are the same. An example of an unweighted edge would be a friendship tie in a social network site. 
Table I. Social Network Types (for person to person unimodal networks)

\begin{tabular}{|l|l|l|l|}
\hline $\begin{array}{l}\text { Direct/ } \\
\text { Indirect }\end{array}$ & Symmetry & $\begin{array}{l}\text { Edge } \\
\text { Weight }\end{array}$ & Examples \\
\hline Direct & Symmetric & Unweighted & Person A \& B are friends or contacts (e.g., social networking friends as in Facebook or LinkedIn) \\
\hline Direct & Symmetric & Weighted & Person A \& B are friends and friendship "strength" (i.e., weight) is measured by number of communications. \\
\hline Direct & Asymmetric & Unweighted & Person A follows person B (e.g., Twitter); Person A rated person B \\
\hline Direct & Asymmetric & Weighted & Person A reads or comments on Person B's content (e.g., blog, forum post). Weight = \# of reads or comments. \\
\hline Indirect & Symmetric & Unweighted & Person A \& B are both members of a group or network \\
\hline Indirect & Symmetric & Weighted & Person A \& B have both edited a document (e.g., wiki page) or visited a location; Weight = \# of edits or visits. \\
\hline Indirect & Asymmetric & Unweighted & Person A joined a group before Person B joined the group \\
\hline Indirect & Asymmetric & Weighted & Person A edited a document before Person B edited the same document \\
\hline
\end{tabular}

Table 1 shows examples of the various combinations of these graph types using unimodal networks that include only people as the nodes. However, these same properties apply to unimodal networks with other entities (e.g., wiki page to wiki page networks), as well as multimodal networks.

\section{Single and Cumulative Networks}

So far, we have focused on networks created by a single technical platform (e.g., a wiki, a social networking site, a discussion forum). It is often useful to analyze these networks because interpretation of the nodes and edges is fairly straightforward. However, enterprises often use many different technical platforms making it possible to create cumulative (i.e., hybrid) networks that describe social ties that are captured from usage of multiple technical platforms. For example, a single network generated from a corporate system with a single login can represent the ties between individuals based on their wiki co-edits, friendships, discussion forum replies, and semantic overlap. Networks where ties can indicate multiple things (e.g., friendship, co-edits) are called multiplex networks.

Multiplex networks add significant complexity to interpretation and analysis as people, threads, blogs, forums, documents, topics and keywords all exist in the same data space. The abundance of related network data makes possible new types of analysis not historically feasible due to the costs of collecting data. The increased analytic complexity may be justified and offset by the added insight into how people are connected to others through different media (i.e. wiki, discussion forum, friends). However, the growing challenge imposed by managing many networks over multiple time slices highlights the need for better tools to support the workflow and management of large social media network datasets.

Existing tools do allow for analysis of these networks, although a bit of extra work is typically needed. One strategy for dealing with cumulative networks is to collapse several networks into one. A directed graph may be used where undirected ties are translated into two directed ties in opposing directions. A network tie could be shown between two people (a) if they have co-edited a wiki page AND are friends, (b) if they have co-edited a wiki page OR are friends, or (c) the weight of the tie could depend on the number of different networks that connect two individuals. Another approach to visually displaying cumulative networks is to show the ties between people (e.g., forum replies) and change the node attributes (e.g., size) based on involvement in another medium (e.g., edits to the wiki). A final approach is to create complementary graphs using similar visual properties and labels to help compare them (as was done in Figures 1 and 2).

\section{ANALYZING ENTERPRISE SOCIAL NETWORKS}

Making sense of the enormous amount of potential data is a central challenge of social network analysis. It is essential that analysts identify their primary goals and research questions in order to select appropriate metrics and visualizations. Some of the most important social network related questions include:

- What kinds of roles are being performed within an enterprise's social media repositories?

- Which individuals play important social roles within an enterprise or organization?

- What subgroups exist? Do connections between organizational divisions exist? Who plays the bridge roles that connect otherwise unconnected groups?

- How do some people convert their contacts to adopt a new technology or practice more than others?

- How do the overall structures of an enterprise's social networks change after a particular event (e.g., a company social; a round of new hires or layoffs)?

Almost all forms of analysis and research questions will require the transformation of event data into network data. For example, logs of who replied to whose message can be transformed into an edge list with the name of a replier to a message in one column and the person she is replying to in the next column. An edge list is a compact representation of a set of relationships that in aggregate forms a network. An alternative representation of a network is an incidence matrix of entities with the strength of their tie at the intersection of the row and column representing people.

With an edge list or matrix representation of a social network in hand, the next step is to calculate a set of descriptive network metrics. These may include the count of nodes, edges, the overall graph density, and for each node its in-degree and out-degree (when the network is directed, otherwise just degree), clustering coefficient, betweenness centrality, closeness centrality, eigenvector centrality, and tie strength. Not all of these measures can be applied to every type of graph. In particular, multimodal graphs require careful interpretation of each term. For example, in Figure 1, the degree for people indicates the number of unique wiki pages they have edited, whereas the degree of each wiki page indicates the number of unique authors of that page. A metric such as betweenness centrality does not have a clear definition in such a network either, since it applies to uni-modal networks. 
Table II. Social Roles and Social Metrics

\begin{tabular}{|c|c|c|c|c|c|c|}
\hline \multirow{15}{*}{ } & & Answer Person & Question Person & $\begin{array}{c}\text { Discussion or } \\
\text { Comment Person } \\
\end{array}$ & Originator & Influencer \\
\hline & Degree (In-Degree + Out-Degree) & High & Low & High & High & Medium \\
\hline & Reply In-Degree & Low & Low & High & High & High \\
\hline & Reply Out-Degree & High & Low & High & Low to Medium & Low \\
\hline & Read In-Degree & High & Low & High & High & High \\
\hline & Read Out-Degree & Medium & Low & High & Low to medium & Low \\
\hline & Betweenness Centrality & High & Low & High & High & High \\
\hline & Neighbors' Mean Degree & Low & High & High & High & High \\
\hline & Closeness Centrality & Low & Low & Low & High & High \\
\hline & Eigenvector Centrality & High & Low & High & High & High \\
\hline & Clustering Coefficient & Low & Low & High & Low to medium & Low \\
\hline & Threads Started & Low & Low & Low & High & Low \\
\hline & Messages Posted & High & Low & High & High & Low \\
\hline & Reply Messages & High & Low & High & Low to medium & Low \\
\hline & Days Active & High & Low & High & High & Low to medium \\
\hline
\end{tabular}

Once a set of social media networks have been constructed and social network measurements have been calculated, the resulting dataset can be used for many applications. For example, they can be used to create reports about community health, comparisons of subgroups, and identification of important individuals, as well as in applications that rank, sort, compare, and search for content and experts.

Sorting and visualizing social media datasets based on network attributes and metrics helps highlight important documents and individuals. Users and their documents, messages, comments, and posts have highly variable network properties. For example, some people have a high degree in their friends graph while others are sparsely connected. Some people are connected to others who are well connected to each other creating a clique, while others are connected to people that are relatively unconnected. Mapping network metrics onto visual properties as we have done in Figures 1 and 2 allows analysts to quickly identify important people, which can then be explored further by looking at their content.

While social network analysis and visualization is largely a manual process due to its complexity, common patterns can be automatically detected to help provide insights into common social practices and roles. New conglomerations of network analysis metrics can help explain many social phenomena including group formation, group cohesion, social roles, personal influence, and community health. Currently, few such aggregate social metrics have been rigorously developed despite increased interest in them from corporations and community and organizational analysts. Here we briefly describe how specific social metrics map to aggregate social roles (i.e., types of participants).

Table II shows various social metrics that describe each node's activity and network position. Five different social roles are described in terms of these social metrics. Each social role can be captured in a composite value that takes various metrics into consideration. Composite values must select the most important social metrics, scale them appropriately, and weigh each individual factor.

An example formula can provide a basic scoring for "discussion people", a type of contributor who contributes heavily to conversations that are often started by others. These participants are often highly connected to other discussion people. Table III shows an example of an aggregate Discussion Person score that can be constructed from six ratios. Each of the terms is a mixture of social metrics, designed to range from 0 to 1 with larger values indicating a higher probability of performing the social role of discussion person.

Table III. Discussion Person Metrics

\begin{tabular}{|l|l|l|}
\hline Term & Value & Explanation \\
\hline Verbosity & $1-$ (Thread count/total posts) & $\begin{array}{l}\text { More messages per thread } \\
\text { is better }\end{array}$ \\
\hline Initiation & $\begin{array}{l}1-(\text { initiated threads/total } \\
\text { threads) }\end{array}$ & $\begin{array}{l}\text { Ego avoids initiating } \\
\text { threads }\end{array}$ \\
\hline Outgoing & Outdegree/degree & Ego replies to many \\
\hline Attractiveness & $\begin{array}{l}\text { Indegree/(count of replying } \\
\text { authors) }\end{array}$ & Many others reply to Ego \\
\hline Connectedness & $1-1 /$ mean(neighbors' degree) & $\begin{array}{l}\text { Ego's alters have high } \\
\text { degree, are highly } \\
\text { connected }\end{array}$ \\
\hline Activity & $\begin{array}{l}\text { (days with a reply post) / } \\
\text { (days since last -first visit) }\end{array}$ & $\begin{array}{l}\text { Ego's participation across } \\
\text { possible days }\end{array}$ \\
\hline
\end{tabular}

As indicated in Table II, combinations of these metrics can be used to identify other social roles as well. Data from other networks (e.g., wikis, social networking sites, semantic terms) could also be used to augment these social roles or help identify roles that span multiple networks. While we have focused on identifying social roles, similar techniques could be used to develop aggregate metrics for a host of other variables of interest such as community health, group cohesion, boundary spanners, etc. Comparing these values across communities, groups, and individuals can help managers, analysts, and individuals know how well they are performing compared to others. It can also help managers know when interventions or education may help improve performance, or help identify successful individuals and groups so best practices can be identified and shared. Tracking metrics over time can help managers measure the effect of interventions.

\section{DISCUSSION \& CONCLUSIONS}

Social media tools provide a wealth of data that can be transformed into insights about the structure and dynamics of an enterprise or organization. These insights can be generated through manual analysis and visualization techniques or captured, albeit imperfectly, through aggregate social metrics. Managers and analysts can use these metrics to better understand organizational dynamics, allowing them to better 
measure the effects of interventions and events. These metrics can also be fed into technical systems like search and sorting and social systems like organizational health monitoring and human resource management. We imagine a future, for example, in which the rate of integration of new employees into an organization's social media practices is measured precisely and reported to all users of a media platform.

These data, over time, will give employees and managers better insight into the empirical patterns of activity and connection within an organization. New paths to prominence will emerge as a result, as new kinds of skills attract more attention in enterprise ranking systems. These measures will make clear the variation between groups in terms of their cohesion and solidarity, help identify individuals who can bridge organizational silos, and bring to light informal networks and communities of practice.

As more organizations and enterprises adopt social media platforms, collections of multiple organization scale data sets will allow for a quantification of the variation among different social media repositories. This will help new managers and employees benchmark their own social media usage against others to identify weaknesses and strengths. Developing a core set of standard social metrics that are widely used to analyze a variety of networks would be invaluable in furthering our understanding of social phenomena.

Despite the potential benefits of analyzing social media networks, there are many potential problems with using them as a basis for mapping actual social relationships. These data confound the use of tools with social relationships. An employee may have a number of friends but if he doesn't like using the social networking site this fact is less apparent to others. If decisions are made based on social network structures (e.g., deciding who to lay-off) then people will have perverse incentives to use these tools - i.e., trying to friend as many people as possible without regard to building actual friendship ties. These problems will diminish as more and more work is performed via these tools, but they will surely persist.

As new social media applications are created, particularly as mobile social software applications and devices become mainstream, new social network datasets become practical to construct. Social network services like Facebook made direct, symmetrical relationship networks very popular. The recent rise of Twitter has focused attention on the creation of direct, asymmetric networks. As location aware devices and other sensors become common on mobile devices, new forms of ties can be detected and aggregated into new forms of social networks [16]. Systems like SpotMe, nTag, and SenseNet illustrate the ways emerging mobile technologies are creating new ways to detect associations between people and places. We hope this paper will help characterize these new networks and provide a general strategy for turning the various types of network data into useful social metrics.

More enterprises will adopt social media platforms, making the analysis of network datasets a mainstream element of many organizations' operations. It is our hope that enterprises will take advantage of these networks to illuminate the internal structures and dynamics of organizations in novel and useful ways and that researchers will support them in these efforts.

\section{ACKNOWLEDGMENTS}

We appreciate the assistance of Telligent Systems, the NodeXL development Team, and the students of social media analysis classes at the University of Maryland.

\section{REFERENCES}

[1] Smith, M., Shneiderman, B., Milic-Frayling, N., Rodrigues, E.M., Barash, V., Dunne, C., Capone, T., Perer, A., Gleave, E. 2009. Analyzing Social (Media) Network Data with NodeXL. In Proceedings of Communities and Technologies (C\&T) (University Park, Pennsylvania, June 25 - 27, 2009). ACM, New York, NY.

[2] Perer, A. and Shneiderman, B. 2008. Integrating statistics and visualization: case studies of gaining clarity during exploratory data analysis. In Proceeding of the Twenty-Sixth Annual SIGCHI Conference on Human Factors in Computing Systems (Florence, Italy, April 05 - 10, 2008). CHI '08. ACM, New York, NY, 265-274. DOI= http://doi.acm.org/10.1145/1357054.1357101

[3] Welser, H., Gleave, E., \& Smith, M. Visualizing the Signatures of Social Roles in Online Discussion Groups. J. Soc. Struct. 8, 2, 2007.

[4] Barash, V., Smith, M., Getoor, L., and Welser, H. T. Distinguishing Knowledge vs. Social Capital in Social Media with Roles and Context. 2009. ICWSM. Association for the Advancement of Artificial Intelligence.

[5] Himelboim, I., Gleave, E., Smith, M. Discussion catalysts in online political discussions: Content importers and conversation starters. Journal of Computer Mediated Communication [Forthcoming].

[6] Burt, R. Structural Holes: The Social Structure of Competition, Harvard University Press, 1995.

[7] Cross, R., Parker, A. The Hidden Power of Social Networks: Understanding How Work Really Gets Done in Organizations, Harvard Business School Press, 2004.

[8] Cross, R. and Thomas, R. J. Driving Results Through Social Networks: How Top Organizations Leverage Networks for Performance and Growth, Jossey-Bass, 2009.

[9] Kilduff, M. and Tsai, W. Social Networks and Organizations, Sage Publications Ltd, 2003.

[10] Scott, J. P. Social Network Analysis: A Handbook, Sage Publications Ltd, 2000.

[11] Perer, A. and Smith, M. A. 2006. Contrasting portraits of email practices: visual approaches to reflection and analysis. In Proceedings of the Working Conference on Advanced Visual interfaces (Venezia, Italy, May 23 - 26, 2006). AVI '06. ACM, New York, NY, 389-395. DOI= http://doi.acm.org/10.1145/1133265.1133346

[12] Bulkley, N. \& Van Alstyne, M.W. An Empirical Analysis of Strategies and Efficiencies in Social Networks. (February 2006). MIT Sloan Research Paper No. 4682-08. Available at SSRN: http://ssrn.com/abstract $=887406$

[13] Aral, S., Brynjolfsson, E. and Van Alstyne, M. W. Information, Technology and Information Worker Productivity. MIT Sloan Research Paper. Available at SSRN: http://ssrn.com/abstract=942310

[14] Viégas, F. B., Golder, S., and Donath, J. 2006. Visualizing email content: portraying relationships from conversational histories. In Proceedings of the SIGCHI Conference on Human Factors in Computing Systems (Montréal, Québec, Canada, April 22 - 27, 2006). R. Grinter, T. Rodden, P. Aoki, E. Cutrell, R. Jeffries, and G. Olson, Eds. CHI '06. ACM, New York, NY, 979-988. DOI= http://doi.acm.org/10.1145/1124772.1124919

[15] Viégas, F. B., Wattenberg, M., and Dave, K. 2004. Studying cooperation and conflict between authors with history flow visualizations. In Proceedings of the SIGCHI Conference on Human Factors in Computing Systems (Vienna, Austria, April 24 - 29, 2004). CHI '04. ACM, New York, NY, 575-582. DOI= http://doi.acm.org/10.1145/985692.985765

[16] Counts, S., Smith, M. Where We Were: Communities for Sharing Space-Time Trails. Proceedings of the 15th annual ACM international symposium on Advances in geographic information systems (2007). 